\title{
Nano-selenium foliar applications enhance the nutrient quality of pepper by activating the capsaicinoid synthetic pathway
}

Dong $\mathrm{Li}^{\mathrm{a}}$, Chunran Zhou ${ }^{\mathrm{a}}$, Jingbang Zhang ${ }^{\mathrm{a}}$, Quanshun $\mathrm{An}^{\mathrm{a}}$, Yangliu Wu ${ }^{\mathrm{a}}$, Jia-Qi Li ${ }^{\mathrm{a}}$ and Canping Pan ${ }^{\mathrm{a}^{*}}$

${ }^{\mathrm{a}}$ Innovation Center of Pesticide Research, Department of Applied Chemistry, College of Science, China

Agricultural University, Beijing 100193, China

* Corresponding authors

Jia-Qi Li: jiaqili@cau.edu.cn

Canping Pan: canpingp@cau.edu.cn

Total number of pages: 8

Total number of figures: 1

Total number of tables: 3 


\section{SUPPORTING TABLES AND FIGURES}

Figure S1. A: Typical TEM image.B: HRTEM of an individual nano-selenium.C: particle size distribution of nano-Se solution

Table S1. HPLC-MS/MS parameters of phytohormones

Table S2. HPLC-MS/MS parameters of capsaicin and dihydrocapsaicin

Table S3. Primer sequences for qPCR 

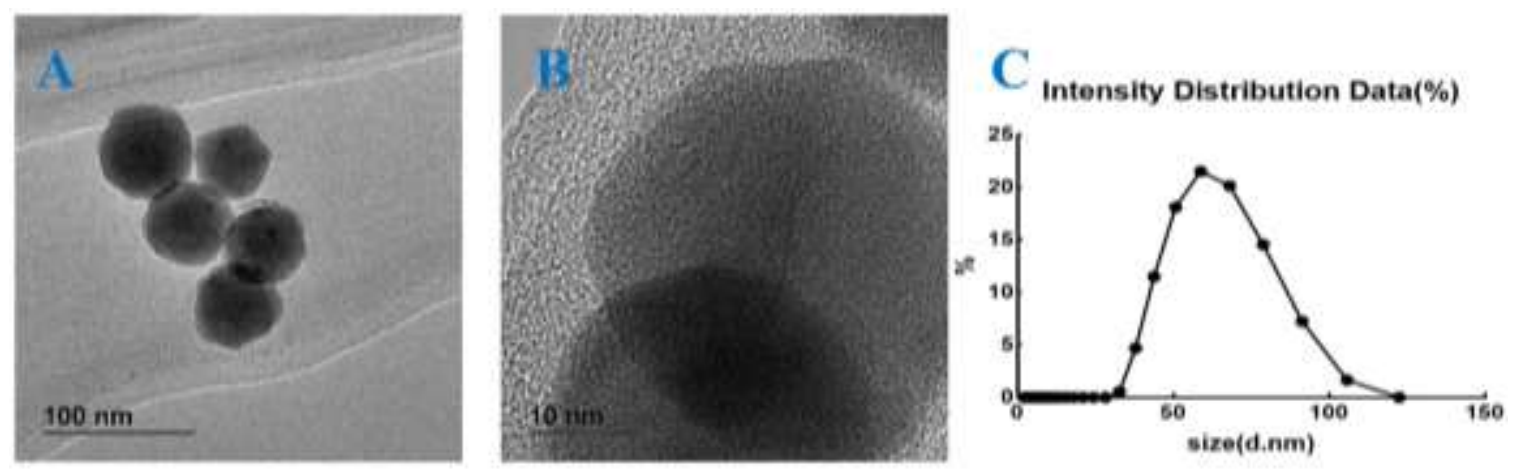

Figure S1. A: Typical TEM image.B: HRTEM of an individual nano-selenium.C: particle size distribution of nano-Se solution 
Table S1. HPLC-MS/MS parameters of phytohormones

\begin{tabular}{|c|c|c|c|c|c|c|}
\hline Name & $\begin{array}{c}\text { Precursor/quantitative } \\
\text { ion }(\mathbf{m} / \mathbf{z})\end{array}$ & $\begin{array}{c}\text { Precursor/qualitative } \\
\text { ion }(\mathbf{m} / \mathbf{z})\end{array}$ & $\begin{array}{l}\text { Retention } \\
\text { time (min) }\end{array}$ & $\begin{array}{l}\text { Collision } \\
\text { energy } \\
\text { (CE) (V) }\end{array}$ & Polarity & $\begin{array}{c}\text { Delta } \\
\text { EMV(-) }\end{array}$ \\
\hline Jasmonic acid & $209.2 / 59.1$ & $209.2 / 209.2$ & 1.81 & $5 / 5$ & Negative & 500 \\
\hline Salicylic acid & $137.1 / 93.1$ & 137.1/137.1 & 1.64 & $5 / 15$ & Negative & 500 \\
\hline Abscisic acid & $263.3 / 153.1$ & $263.3 / 201.0$ & 1.75 & $5 / 5$ & Negative & 500 \\
\hline
\end{tabular}


Table S2. HPLC-MS/MS parameters of capsaicin and dihydrocapsaicin

\begin{tabular}{|c|c|c|c|c|c|c|}
\hline Name & $\begin{array}{c}\text { Precursor/quantitative } \\
\text { ion }(\mathbf{m} / \mathbf{z})\end{array}$ & $\begin{array}{c}\text { Precursor/qualitative } \\
\text { ion }(\mathbf{m} / \mathbf{z})\end{array}$ & $\begin{array}{l}\text { Retention } \\
\text { time } \\
\text { (min) }\end{array}$ & $\begin{array}{l}\text { Collision } \\
\text { energy } \\
\text { (CE) (V) }\end{array}$ & Polarity & $\begin{array}{l}\text { Delta } \\
\text { EMV(-) }\end{array}$ \\
\hline Capsaicin & $306.0 / 137.1$ & $306.0 / 122$ & 1.26 & $15 / 15$ & Positive & 500 \\
\hline Dihydrocapsaicin & $308.2 / 137.1$ & $308.2 / 122$ & 3.78 & $15 / 15$ & Positive & 500 \\
\hline
\end{tabular}


Table S3. Primer sequences for $\mathrm{qPCR}^{1-3}$

\begin{tabular}{|c|c|c|}
\hline Gene & Abbreviation & Sequence \\
\hline Lipoxygenase 2 & 13-LOX2 & $\begin{array}{l}\text { Forward: GGACCGGCGATGCAGAGA } \\
\text { Reverse: ATGTACTTGCATCTTAA }\end{array}$ \\
\hline Lipoxygenase 6 & 13-LOX6 & $\begin{array}{l}\text { Forward: ACTTGGCAGCATGCAATTTGT } \\
\text { Reverse: CGCGTATCTTCTTCGAAAGAC }\end{array}$ \\
\hline Lypoxygenase 3 & LOX3 & $\begin{array}{l}\text { Forward: TGGTGATCCTGCGAATGGTT } \\
\text { Reverse: CGTCCCAATCAAACGTGACA }\end{array}$ \\
\hline Allene oxide cyclase & $\mathrm{AOC}$ & $\begin{array}{l}\text { Forward: AGGGGTTTCCGGTCAAGTA } \\
\text { Reverse: GACCCCAACAAAAGTCGTGA }\end{array}$ \\
\hline Allene oxide synthase & AOS & $\begin{array}{l}\text { Forward: CCGTTCTGTCATTTCATCCA } \\
\text { Reverse: TTCAATGCGGAGAGATTCGT }\end{array}$ \\
\hline Isochorismate synthase & ICS1 & $\begin{array}{l}\text { Forward: TCATCGGTGCACCCAACTC } \\
\text { Reverse: CCAAGACCCTTTTCAACCAA }\end{array}$ \\
\hline Phenylalanine ammonia-lyase 1 & PAL1 & $\begin{array}{l}\text { Forward: GGAAGGAACTTGGAACTGA } \\
\text { Reverse: GCACTTGACAAGCACTAACA }\end{array}$ \\
\hline Phenylalanine ammonia-lyase 2 & PAL2 & $\begin{array}{l}\text { Forward: GGCAAGTCATCCATTCCTAA } \\
\text { Reverse: GGGGACGAGATGAACCAT }\end{array}$ \\
\hline Phenylalanine ammonia-lyase 3 & PAL3 & $\begin{array}{l}\text { Forward: AGGTGAAAGAGTCCGATCA } \\
\text { Reverse: GAATCTGAAAAATGGCACTA }\end{array}$ \\
\hline 9-cis-epoxycarotenoid dioxygenase 1 & NCDE1 & $\begin{array}{l}\text { Forward: GAAAAGGAATGGAAATCGGA } \\
\text { Reverse: CGGGGACGTATATTCTAAAC }\end{array}$ \\
\hline 9-cis-epoxycarotenoid dioxygenase 2 & NCDE2 & $\begin{array}{l}\text { Forward: AGTACCACCAAAAATTATAGG } \\
\text { Reverse: TCTGAGCTTTCCGAATC }\end{array}$ \\
\hline
\end{tabular}




\begin{tabular}{|c|c|c|}
\hline 9-cis-epoxycarotenoid dioxygenase 3 & NCDE3 & $\begin{array}{l}\text { Forward: AAAGAGTGCCATATGGTT } \\
\text { Reverse: TCAAGAAGGGTATTCAATAGA }\end{array}$ \\
\hline Phe ammonia lyase & Pal & $\begin{array}{l}\text { Forward:ATTCGCGCTGCAACTAAGAT } \\
\text { Reverse:CACCGTGTAAGGCCTTGTTT }\end{array}$ \\
\hline Cinnamic acid 4-hydroxylase & $\mathrm{Ca} 4 \mathrm{H}$ & $\begin{array}{l}\text { Forward:CTTGGTTAACGCTTGGTGGT } \\
\text { Reverse:CCGAATGGAAGGAATCTGAA }\end{array}$ \\
\hline 4-coumarate-CoA ligase & $4 \mathrm{CL}$ & $\begin{array}{l}\text { Forward:GGACCGATTGAAGGAATTGA } \\
\text { Reverse:GGACAACAGCAGCATCAGAA }\end{array}$ \\
\hline Coumaric acid 3-hydroxylase & $\mathrm{C} 3 \mathrm{H}$ & $\begin{array}{l}\text { Forward:GCCATCTTCTGCACCATTTT } \\
\text { Reverse:GGCCTGTAATGGAGTCCTCA }\end{array}$ \\
\hline Hydroxycinnamoyl transferase & HCT & $\begin{array}{l}\text { Forward: ATGCAGGGATGAAGATGGAC } \\
\text { Reverse: TAATCAACGGCCGGAATAAG }\end{array}$ \\
\hline Caffeic acid O-methyltransferase & Comt & $\begin{array}{l}\text { Forward: CCTGCGAATGGAAAAGTGAT } \\
\text { Reverse: TCTTTGCCTCCTGGGTTATG }\end{array}$ \\
\hline Aminotransferase & pAmt & $\begin{array}{l}\text { Forward:TGGCAGAGTGATATGGAACC } \\
\text { Reverse:GCGCACCATAAACCAGATAA }\end{array}$ \\
\hline $\begin{array}{l}\text { Branched-chain amino } \\
\text { acid transferase }\end{array}$ & BCAT & $\begin{array}{l}\text { Forward:CAAGGAAGGAACAGCACCAT } \\
\text { Reverse: TCGCCTTTGCTTTCTTCATC }\end{array}$ \\
\hline $\begin{array}{l}\text { Branched-chain a-ketoacid } \\
\text { dehydrogenase }\end{array}$ & BCKDH & $\begin{array}{l}\text { Forward:CGGATGGCTGTTGAAGAAGT } \\
\text { Reverse:CTCCTTTGCAGCTTCTACGC }\end{array}$ \\
\hline$\beta$-Ketoacyl-ACP synthase & Kas & $\begin{array}{l}\text { Forward:GAAAGAATGATCGTGCTTG } \\
\text { Reverse:GTTGGCCAAGGTTAGCATCT }\end{array}$ \\
\hline Acyl carrier protein & Acl & $\begin{array}{l}\text { Forward:TTCCTTCAAGCACAACCAGA } \\
\text { Reverse:GCGAGTAGCTGGCTTCATTC }\end{array}$ \\
\hline Acyl-ACP thioesterase & Fat & $\begin{array}{l}\text { Forward:TGATGGATTTGCGACTACCC } \\
\text { Reverse:CCCAATCCTGCCTTCACTTT }\end{array}$ \\
\hline Acyl-CoA synthetase & Acs & $\begin{array}{l}\text { Forward:TGGCTCAGCTGAATTTGTTG } \\
\text { Reverse:TAACCCGTGAACGTGAAACA }\end{array}$ \\
\hline Acyltransferase & AT3 & $\begin{array}{l}\text { Forward:AGAAGGGAAACTGCCATTTG } \\
\text { Reverse:TCTTTCAGGTCTTCCCCATC }\end{array}$ \\
\hline
\end{tabular}




\section{SUPPORTING REFERENCES}

1.Arce-Rodríguez, M. L.; Ochoa-Alejo, N., An R2R3-MYB Transcription Factor Regulates Capsaicinoid Biosynthesis. Plant Physiology 2017, 174, 1359-1370.

2.Dziurka, M.; Janeczko, A.; Juhász, C.; Gullner, G.; Oklestková, J.; Novák, O.; Saja, D.;

Skoczowski, A.; Tóbiás, I.; Barna, B., Local and systemic hormonal responses in pepper leaves during compatible and incompatible pepper-tobamovirus interactions. Plant Physiology and Biochemistry 2016, 109, 355-364.

3.Mishra, R.; Nanda, S.; Rout, E.; Chand, S. K.; Mohanty, J. N.; Joshi, R. K., Differential expression of defense-related genes in chilli pepper infected with anthracnose pathogen Colletotrichum truncatum. Physiological and Molecular Plant Pathology 2017, 97, 1-10. 